\title{
Maternal High-Fiber Diet Protects Offspring against Type 2 Diabetes
}

\author{
Huishi Toh ${ }^{1, *}$, James A. Thomson ${ }^{2,3,4}$ and Peng Jiang ${ }^{4, *}$ \\ 1 Neuroscience Research Institute, University of California Santa Barbara, Santa Barbara, CA 93117, USA \\ 2 Department of Molecular, Cellular and Developmental Biology, University of California Santa Barbara, \\ Santa Barbara, CA 93106, USA; JThomson@morgridge.org \\ 3 Department of Cell and Regenerative Biology, University of Wisconsin School of Medicine and Public Health, \\ Madison, WI 53726, USA \\ 4 Regenerative Biology Laboratory, Morgridge Institute for Research, Madison, WI 53715, USA \\ * Correspondence: toh@ucsb.edu (H.T.); PJiang@morgridge.org (P.J.)
}

Citation: Toh, H.; Thomson, J.A.; Jiang, P. Maternal High-Fiber Diet Protects Offspring against Type 2 Diabetes. Nutrients 2021, 13, 94. https://doi.org/10.3390/nu13010094

Received: 23 November 2020 Accepted: 28 December 2020 Published: 30 December 2020

Publisher's Note: MDPI stays neutral with regard to jurisdictional clai$\mathrm{ms}$ in published maps and institutional affiliations.

Copyright: (C) 2020 by the authors. Licensee MDPI, Basel, Switzerland. This article is an open access article distributed under the terms and conditions of the Creative Commons Attribution (CC BY) license (https:// creativecommons.org/licenses/by/ $4.0 /)$.

\begin{abstract}
Previous studies have reported that maternal malnutrition is linked to increased risk of developing type 2 diabetes in adulthood. Although several diabetic risk factors associated with early-life environment have been identified, protective factors remain elusive. Here, we conducted a longitudinal study with 671 Nile rats whereby we examined the interplay between early-life environment (maternal diet) and later-life environment (offspring diet) using opposing diets that induce or prevent diet-induced diabetes. Specifically, we modulated the early-life environment throughout oogenesis, pregnancy, and nursing by feeding Nile rat dams a lifelong high-fiber diet to investigate whether the offspring are protected from type 2 diabetes. We found that exposure to a high-fiber maternal diet prior to weaning significantly lowered the risk of diet-induced diabetes in the offspring. Interestingly, offspring consuming a high-fiber diet after weaning did not develop diet-induced diabetes, even when exposed to a diabetogenic maternal diet. Here, we provide the first evidence that the protective effect of a high-fiber diet can be transmitted to the offspring through the maternal diet, which has important implications in diabetes prevention.
\end{abstract}

Keywords: maternal diet; fiber; protection; offspring; hiperglycemia

\section{Introduction}

Diabetes is one of the fastest-growing health challenges facing us today. In the last 20 years, the number of people living with diabetes increased from 151 million to over 463 million [1]; of those, 90-95\% have type 2 diabetes. Type 2 diabetes is a complex disease that is poorly understood. It is characterized by insulin resistance exacerbated by beta cell dysfunction. Lifestyle intervention and current medications including insulin therapy can help regulate blood glucose levels, but in the vast majority of patients, these regimens ultimately fail. Consequently, even with treatment, diabetic patients continue to be at risk for numerous debilitating complications and have approximately a two-fold increased mortality compared to people without diabetes [2]. Hence, prevention of diabetes is a top public health priority around the world.

Diabetic risk is strongly associated with both parental history and eating habits. The odds of developing type 2 diabetes are three- to six-fold higher for adults with at least one diabetic parent compared to adults without diabetic parents [3]. However, genomewide association studies repeatedly reveal that genetic factors insufficiently explain the observed familial aggregation [4,5], suggesting that familial risk is also modulated by non-genetic factors, which might include learned eating behaviors [6,7] as well as exposure to early-life environment [8]. The culpability of diet is well-known but not well-defined. All three classes of macronutrients - carbohydrate, fat, and protein-have been examined quantitatively and qualitatively in the context of type 2 diabetes, but consensus is sorely 
lacking. In general, newer studies have proposed that a low-carbohydrate diet could be more effective for managing type 2 diabetes compared to a low-fat diet $[9,10]$. However, fiber is an exception. Unlike simple carbohydrates, fiber is a member of the carbohydrate food group that is associated with a reduced risk of developing type 2 diabetes [11].

Low birth and one-year-old weights are strongly linked to type 2 diabetes later in life [12]. Based on this observation, Hales and Barker developed the thrifty phenotype hypothesis, which states that poor nutrition during early developmental stages may result in reduced growth of beta cells, resulting in a physiology well-adapted to poor nutrition in later life. However, a switch to improved nutrition in such individuals would lead to the development of type 2 diabetes [13]. Evidence supporting the thrifty phenotype hypothesis comes from examining the offspring of pregnant mothers during the Dutch famine (maladapted to improved nourishment) and the Siege of Leningrad (correctly adapted to malnourishment) [14]. A decade later, Bateson and Gluckman further elaborated on this phenomenon using the predictive adaptive response hypothesis, where the phenotypic plasticity during early development confers an advantage if the future environment matches the predicted environment based on early life exposures [15]. Previous experimental animal studies have similarly demonstrated adverse long-term effects using a maternal malnourished model, frequently using a low-protein (in association with higher fat or carbohydrate) diet during gestation and lactation in sheep, pigs, and rodents [16-19]. However, research to date has focused on the impact of maternal diet in increasing disease susceptibility rather than decreasing the susceptibility to type 2 diabetes.

Nile rats (Arvicanthis niloticus), native to tropical Africa, have a diet primarily of leaves and stems supplemented with insects, seeds, and fruits. The diabetic Nile rat model presents a similar nutritional etiology and disease progression to type 2 diabetes in humans [20-23], and thus it is an exemplary model to study how nutritional imbalances contribute to the development of type 2 diabetes. In a laboratory environment, Nile rats readily develop diabetes when fed a standard rodent chow [22], thereby referred to as a diabetogenic diet (specifically in the context of the Nile rat). Conversely, they do not develop diabetes when fed a high-fiber diet. Both diets used in our study were commercial diets based on their robustness to induce and prevent diet-induced diabetes in the Nile rat.

We conducted a longitudinal study of 671 Nile rats to explore the consequences of two opposing maternal diets on the subsequent susceptibility of type 2 diabetes in the offspring. To avoid potential confounding effects of regularly fasting our animals in a long-term study, we used random blood glucose (RBG) measurements to assess the development and progression of diabetes. Previous studies have compared different measurements for diabetes assessment (RBG, fasting blood glucose, and oral glucose tolerance test) in the Nile rat and demonstrated that RBG is the most reliable early biomarker and a preferred clinical parameter for predicting both incidence of diabetes and its severity in the Nile rat [24].

In the lifelong susceptibility to type 2 diabetes, both exposure to maternal nutrition and self-acquired nutrition act in concert. Previous experimental studies have investigated maternal diet as an isolated variable [25,26], but failed to incorporate offspring diet as a covariate. Additionally, we have included sex as a biological variable, acknowledging the presence of sexual dimorphism in diet-induced type 2 diabetes $[27,28]$. Using a simple Bayesian network, we incorporated three factors-maternal diet, offspring diet, and sex-in our statistical model to examine the probability of diabetes caused by each factor. Our aims are to provide a definitive study of how maternal diet (early-life environment) juxtaposed with offspring diet (later-life environment) shifts the long-term risk susceptibility and to clarify the role of maternal diet in the prevention of type 2 diabetes.

\section{Materials and Methods}

\subsection{Animals}

All animal experiments were approved by the University of California Santa Barbara, Institutional Animal Care and Use Committee, and conducted in accord with the NIH 
Guide for the Care and Use of Laboratory Animals. Our founder Nile rats were derived from the Brandeis University colony of the KC Hayes Laboratory. Nile rats in UCSB are housed at $21-26^{\circ} \mathrm{C}$ in a conventional facility with individually ventilated cages and are provided autoclaved Sanichips as bedding material.

\subsection{Ethical Statement}

The Institutional Animal Care and Use Committee of the University of California Santa Barbara approved the study protocol (ID: 893; 12/03/2014) in accordance with the NIH Guide for the Care and Use of Laboratory Animals. All applicable international, national, and institutional guidelines for the care and use of animals were followed.

\subsection{Experimental Animal Protocol}

Nile rat dams were randomly fed a high-fiber diet (Lab Diet 5L3M; Newco Specialty, Rancho Cucamonga, CA, USA) or a diabetogenic diet (Formulab Diet 5008; Newco Specialty, Rancho Cucamonga, CA, USA) [22]. The percentage of crude fiber was $23 \%$ for the highfiber diet and only $4 \%$ for the diabetogenic diet. The ratios for the percentage of calories provided by carbohydrate, fat, and protein were 67:10:23 for the high-fiber diet and 56:17:27 for the diabetogenic diet. Littermates were randomly assigned to either the high- fiber or diabetogenic diet, which was maintained throughout the study. Using sibling-matched diet assignments allowed us to redistribute possible genetic bias into each of the high-fiber or diabetogenic diet groups. Random blood glucose (RBG) levels were measured every four weeks starting at weaning age (4 weeks old). Diabetic Nile rats were defined as having RBG $>100$ based on prior studies [24]. To reduce adverse events from diabetic complications, Nile rats with RBG $>500$ were euthanized. The number of Nile rats grouped by diet scheme and sex is provided in Supplementary Figure S1, and details from each Nile rat are provided in Supplementary Table S1. In a laboratory environment, Nile rats readily develop diabetes when fed a standard rodent chow [22], thereby referred to as a diabetogenic diet (specifically in the context of the Nile rat). Conversely, they do not develop diabetes when fed a high-fiber diet. Both diets used were commercial diets based on their robustness to induce and prevent diet-induced diabetes in the Nile rat.

\subsection{Modeling the Probability of Diabetes}

We used a simple Bayesian model framework [29] consisting of three variables (maternal diet, offspring diet, and sex) to model the probability of diabetes from 4 to 40 weeks at 4-week intervals (Supplementary Figure S2). The Bayesian network parameter learning and probability inference were implemented by R package (“bnlearn”) [30].

\subsection{Estimating Human Equivalent Age of Rodents}

To provide human relevance, we presented in parallel the human equivalent age of rodents obtained from the Jackson Laboratory (life phase equivalencies between human and mouse) [31].

\section{Results}

3.1. Offspring on Diabetogenic Diet were Protected against Diabetes when Mothers Consumed A High-Fiber Diet

We observed a significant difference between the diabetogenic diet offspring from mothers that consumed a high-fiber diet compared to a diabetogenic diet. As shown in Figure 1, in the group where offspring and mothers shared the same diabetogenic diets, the average RBG depicted an upward trend from 4 weeks old and clearly reflected a diabetic value from 12 weeks old. The upward trend continued until around 36 weeks old, when the average RBG plateaued at around $300 \mathrm{mg} / \mathrm{dL}$. In contrast, in the group with mothers on a high-fiber diet, the average RBG wavered around $100 \mathrm{mg} / \mathrm{dL}$ and did not reach a diabetic value until around 28 weeks, suggesting a delay in the onset of diabetes of about 16 weeks. Furthermore, with each subsequent reading at a later timepoint, the difference between the 
diabetogenic maternal diet group and the high-fiber maternal diet group became larger, suggesting that, in addition to the delayed onset of hyperglycemia, the progression of diabetes was additionally slowed down.

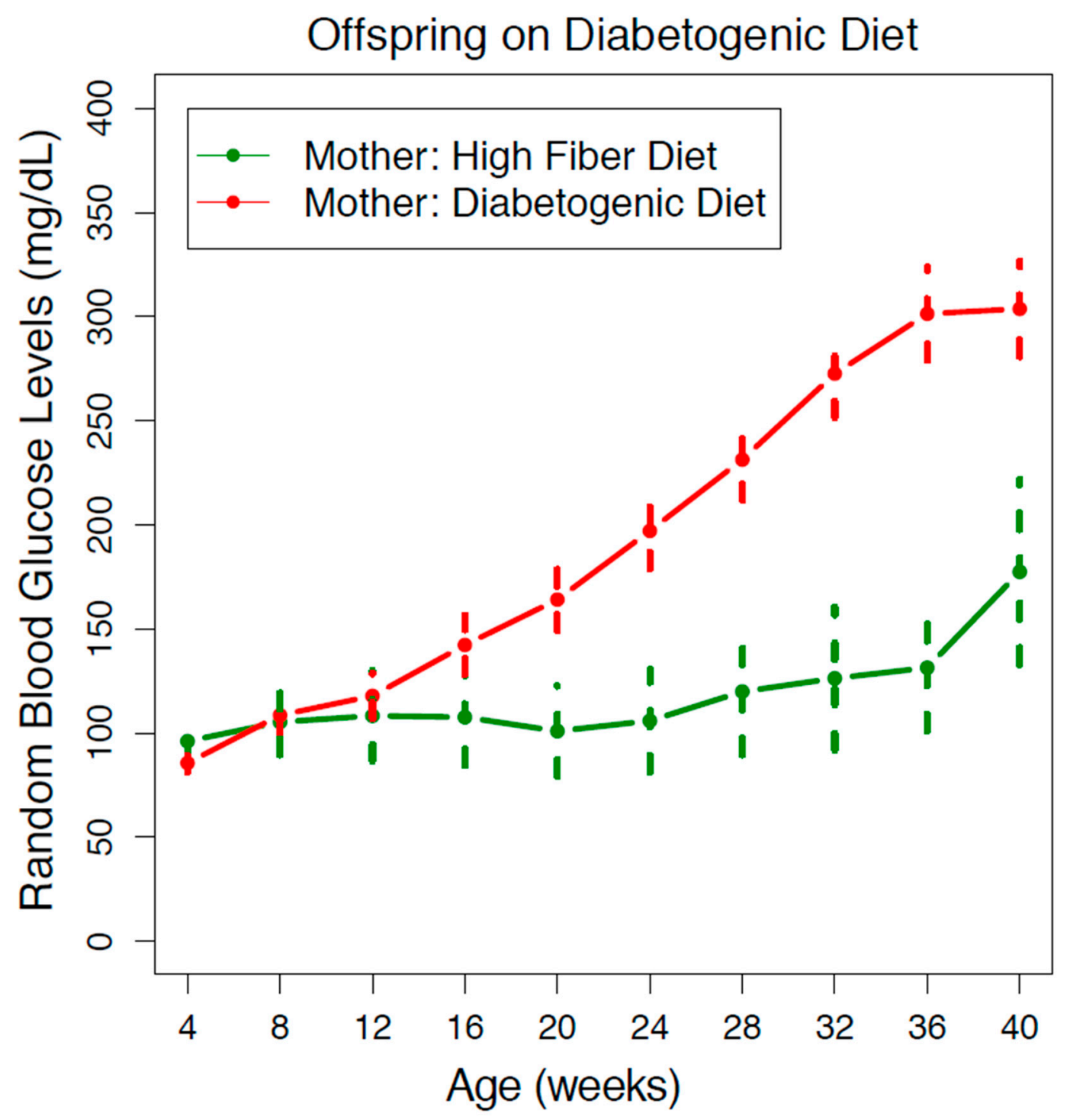

Figure 1. Maternal high-fiber diet protects offspring on diabetogenic diet. The progression of diabetes in the Nile rat offspring (both sexes) fed a diabetogenic diet is depicted as a series of random blood glucose levels from 4 to 40 weeks old. The offspring of mothers with a high-fiber diet, on average, experienced a delayed onset of diabetes and progressed more gradually to higher levels of RBG compared to offspring of mothers with a diabetogenic diet. The error bars reflect a $95 \%$ confidence interval of the data. The number of Nile rats: $n=95$ (green); $n=335$ (red).

\subsection{Offspring on A High-Fiber Diet did not Develop Diabetes even when Mothers Consumed A Diabetogenic Diet}

Interestingly, a high-fiber diet conferred long-term protection to offspring against diabetes regardless of maternal diet. In offspring that were fed a high-fiber diet after weaning, the average blood glucose trajectory between those that were from mothers with a high-fiber diet or those that were from mothers with a diabetogenic diet was similar. The average RBG in both groups never reached the diabetic threshold of RBG > $100 \mathrm{mg} / \mathrm{dL}$, from 4 to 40 weeks old (Figure 2). This pattern was observed in both sexes (Supplementary Figure S3). 


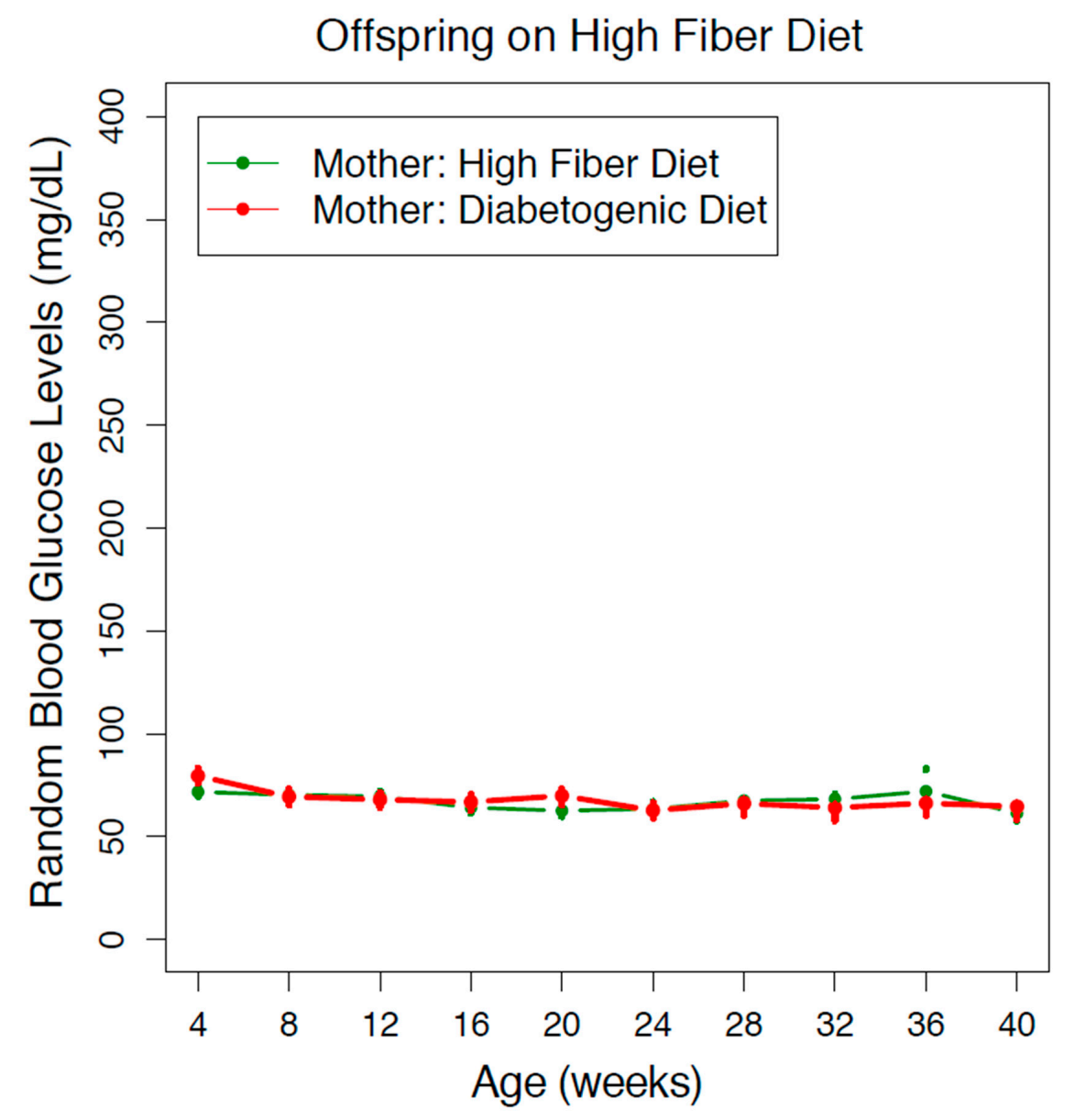

Figure 2. Maternal diet does not influence offspring on a high-fiber diet. The progression of diabetes in the Nile rat offspring (both sexes) fed a high-fiber diet is depicted as a series of random blood glucose levels from 4 to 40 weeks old. When the offspring were fed a high-fiber diet, they did not develop diabetes from mothers with a high-fiber diet or from mothers with a diabetogenic diet. The error bars reflect a 95\% confidence interval of the data. The number of Nile rats: $n=131$ (green), $n=90$ (red).

\subsection{Protective Effect of the High-Fiber Maternal Diet was more Pronounced in Males}

The blood glucose lowering effect of the maternal high-fiber diet on diabetogenic diet offspring was observed in both sexes. Males benefitted more because they had a more severe hyperglycemic profile compared to the females when on a diabetogenic diet. However, the maternal high-fiber diet protection lowered the mean RBG levels to a similar normal level in both male and female offspring (Figure 3).

\subsection{High-Fiber Maternal Diet Reduced the Long-Term Risk Susceptibility of Diet-Induced Diabetes}

In our experimental design, we have three factors-maternal diet, offspring diet, and sex-that affect the risk susceptibility of diet-induced diabetes. To account for all three factors, we built a statistical model using a simple Bayesian network from 671 Nile rats with RBG values ranging from 4 to 40 weeks old. From our model, we calculated the probability of diabetes in diabetogenic diet offspring within the high-fiber maternal diet group compared to the diabetogenic maternal diet group. As shown in Figure 4, the probability of diabetes in the diabetogenic maternal diet group increased from $28.74 \%$ at 12 weeks old (equivalent to 20 years old in a human) to $81.77 \%$ at 36 weeks old (equivalent to 38 years old in a human). In comparison, the high-fiber maternal diet group increased from $13.21 \%$ at 12 weeks old to $32.87 \%$ at 36 weeks old. These data are replotted according to sex in Figure 5. 

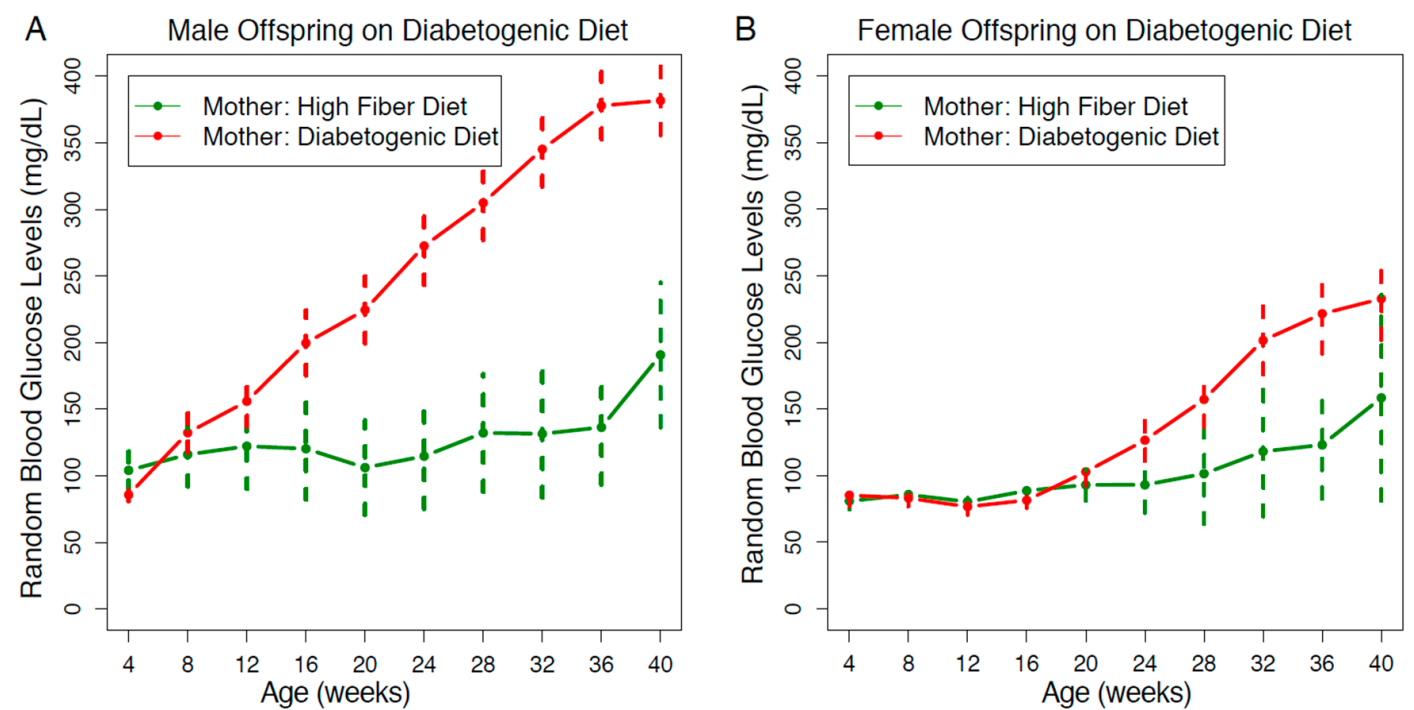

Figure 3. The decrease in RBG trajectory from a maternal high-fiber diet was more obvious in the male offspring. (A) In male offspring on a diabetogenic diet, the protective effect from a maternal high-fiber diet was evident from 12 to 40 weeks old. The number of Nile rats: $n=62$ (green), $n=183$ (red). (B) In female offspring on a diabetogenic diet, the protective effect from a maternal high-fiber diet was evident from 24 to 40 weeks old. The error bars reflect a $95 \%$ confidence interval of the data. The number of Nile rats: $n=33$ (green), $n=172$ (red).

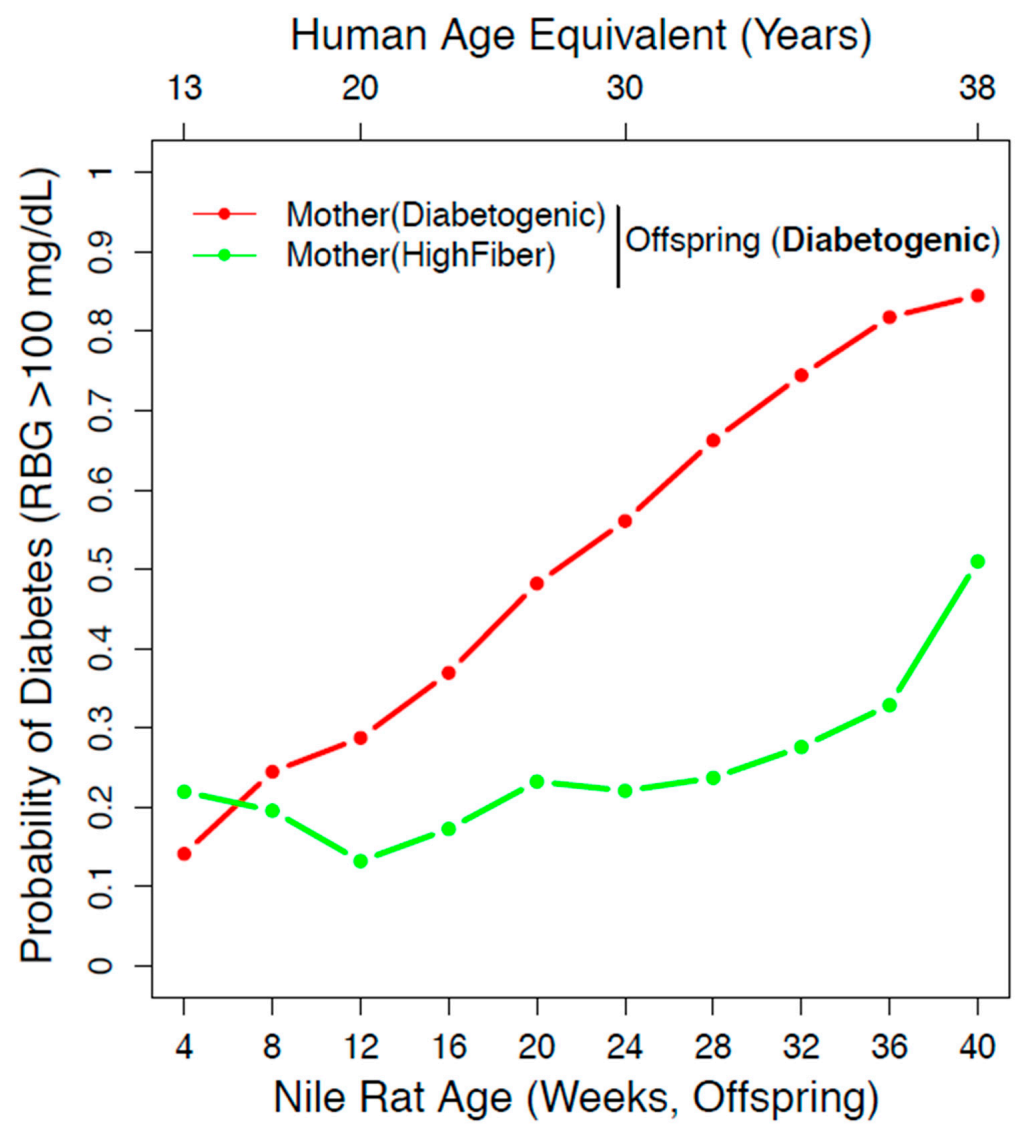

Figure 4. Offspring have a reduced probability of diabetes when mothers consumed a high-fiber diet. We used a simple Bayesian network that incorporated maternal diet, offspring diet, and sex to calculate the probability of diabetes due to maternal diet differences. When the offspring is on a diabetogenic diet, the probability of diabetes is greatly reduced in the group with a maternal high-fiber diet compared to a maternal diabetogenic diet. The number of Nile rats: $n=95$ (green), $n=355$ (red). 

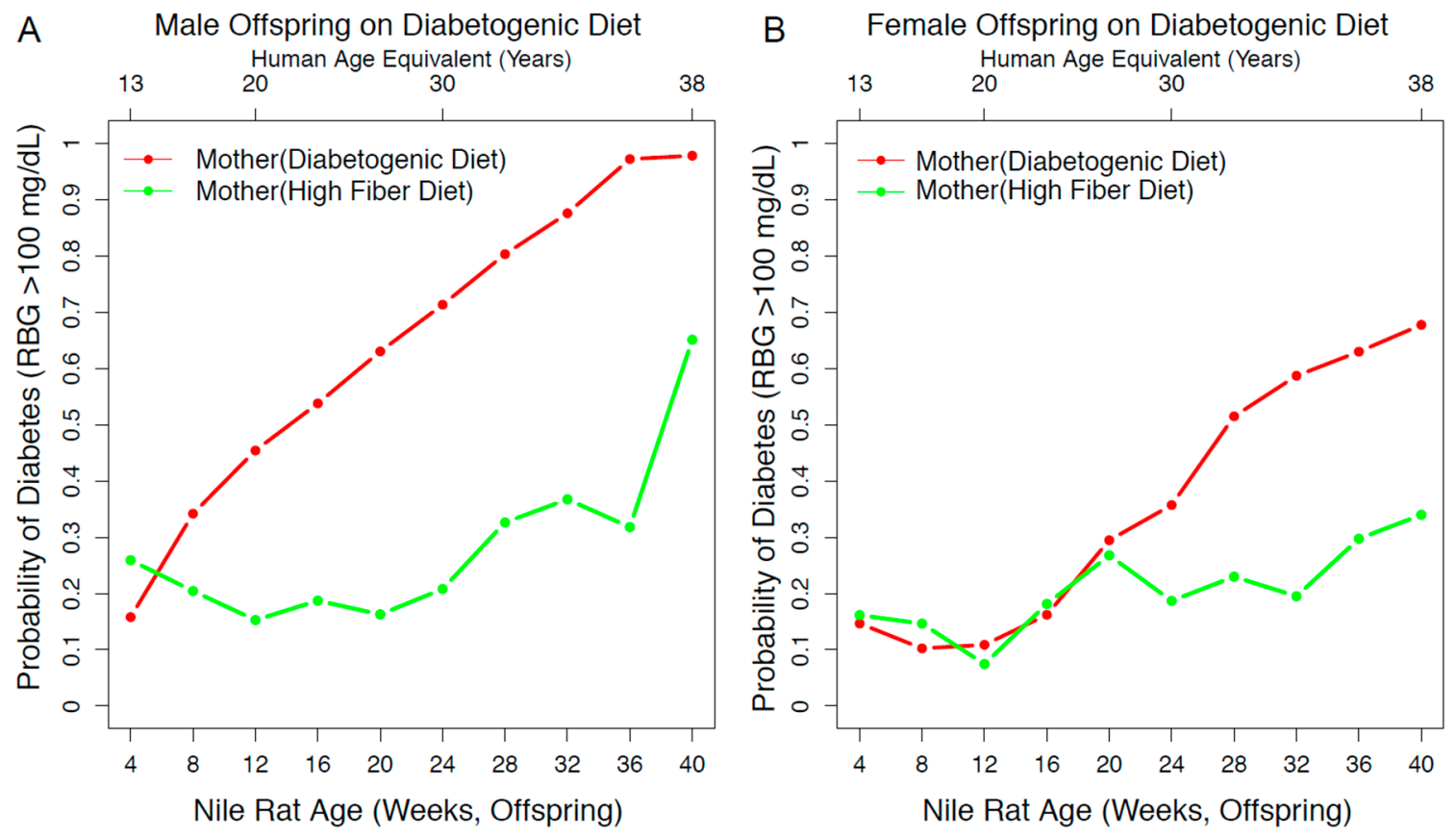

Figure 5. The probability of diabetes due to maternal diet was reduced to a larger extent in the male offspring. (A) In male offspring on a diabetogenic diet, the ones from mothers on a high-fiber diet experienced a reduced probability of diabetes from 8 to 40 weeks old. The number of Nile rats: $n=62$ (green), $n=183$ (red). (B) In female offspring on a diabetogenic diet, the ones from mothers on a high-fiber diet experienced a reduced probability from 25 to 40 weeks old. The number of Nile rats: $n=33$ (green), $n=172$ (red).

\section{Discussion}

Effective prevention measures are needed to curb the steep rising rate of type 2 diabetes. Susceptibility to type 2 diabetes is subjected to cumulative events throughout life, possibly starting from conception, described as a life-course perspective. Early-life development in utero and during infancy has been identified as a sensitive period of phenotypic plasticity where maternal nutrition can impact the risk susceptibility of type 2 diabetes in later life. Thus, a better understanding of how maternal diet modulates disease susceptibility would inform us on effective ways to prevent type 2 diabetes.

Here, we use two opposing diets that either induce or prevent diabetes in the Nile rat to understand how maternal nutrition shifts the disease risk of type 2 diabetes. Our experimental design is different in a few ways. First, we used a lifelong maternal diet in the Nile rat dams. In other experimental animal studies, the maternal diet is typically restricted to pregnancy and lactation. However, the influence of maternal diet could include the period of oogenesis that occurs during preconception. We also considered the possibility that abruptly switching diets just prior to conception may trigger altered behavioral changes due to interrupted eating habits not pertinent to maternal nutrition. Second, maternal diet and offspring diet were treated as two independent variables, providing us an opportunity to observe the interplay between early-life nutrition prior to weaning and later-life nutrition after weaning. This added layer of information cannot be obtained from studies using maternal diet as the sole experimental variable. Additionally, we considered sex as a biological variable, versus the majority of diabetic studies in animal models where only males are represented. Third, the risk susceptibility of diet-induced diabetes was analyzed in a longitudinal manner. Diet-induced diabetes has a complicated disease profile where onset and severity are highly variable, both in the human population and the Nile rat model. Thus, we generated a detailed longitudinal dataset to capture dynamic changes over time. Additionally, our dataset for this study is unusually large, consisting of RBG 
values from 671 Nile rats from 4 to 40 weeks old. This allows us to validate our findings using a robust Bayesian model with three nodes: maternal diet, offspring diet, and sex.

We found that a maternal high-fiber diet reduced the probability of diabetes in offspring that consumed a diabetogenic diet after weaning (Figure 4). Although the ratios of macronutrients were not dramatically different, the percentage of crude fiber was $23 \%$ for the high-fiber diet and only $4 \%$ for the diabetogenic diet. The beneficial effect of fiber on diet-induced diabetes in the Nile rat had previously been demonstrated by Bolsinger [24]. Prospective cohort studies like the Stockholm Diabetes Prevention Program study have found that consumption of whole grain protects against the development of prediabetes and type 2 diabetes [32]. Additionally, a recent epidemiological meta-analysis reported a $10-20 \%$ reduction in type 2 diabetes incidence associated with higher dietary fiber using data from 48,468 people in 17 studies [33]. The observed protective effect could be due to the presence of dietary fiber that promotes an anti-diabetic microbiome by increasing the amount of short-chain fatty acids [34]. Another possibility is that fiber reduces the intake of calories, and the high-fiber diet is essentially a low-calorie diet. Future studies using purified diets can be used to assess how dietary fiber confers protection against type 2 diabetes. Additionally, offspring from mothers on diabetogenic diets are, on average, 1.5-fold heavier than offspring from mothers on high-fiber diets at the time of weaning (Supplementary Figure S4). Perhaps a maternal high-fiber diet protects offspring from diet-induced diabetes by controlling weight gain during early development. Whereas Nile rat dams on high-fiber diets never developed diet-induced diabetes, $28.3 \%$ of Nile rat dams on a diabetogenic diet were diabetic prior to pregnancy, and this may additionally impact the offspring from mothers on diabetogenic diets.

The focus of maternal diet (marker of early-life environment) stems from studies on adults that lived through famines where low birth weight was consistently associated with detrimental metabolic health consequences in adult life [35]. To explain these observations, the thrifty phenotype hypothesis and the predictive adaptive response hypothesis were proposed, where a mismatch between early-life environment and later-life environment resulted in increased susceptibility to type 2 diabetes. However, whether it is the mismatched element or the nature of the environmental factor(s) is still controversial. A recent human study investigating different combinations of early- and later-life environmental conditions revealed that adverse early-life conditions were detrimental to later health across all environments, not only in mismatched situations [36]. Our two-by-two factorial design provided two groups with mismatched diets and two groups with matched diets. We found that Nile rats with the high-fiber maternal diet and diabetogenic offspring diet had a diabetes profile that was better than Nile rats with matched diabetogenic diets but worse than Nile rats with matched high-fiber diets. Furthermore, Nile rats with the diabetogenic maternal diet and high-fiber offspring diet did not get diabetes, similar to Nile rats with matched high-fiber diets. Therefore, our findings suggest that it is not the mismatched element that leads to type 2 diabetes; rather, it depends on the nature of the environmental cue. In the Nile rat model, a high-fiber diet is beneficial to prevent diet-induced diabetes whether exposed to it through maternal feeding or consumed later in life.

It would be interesting to see if the protective effect of a maternal high-fiber diet can be observed in other models of type 2 diabetes and the human population. Given the species difference, we expect differences in the susceptibility of diet-induced diabetes, but we postulate that the overall trends in our major findings should be robust regardless of species. One limitation of this study is that we did not record the daily food consumption and calculate the fiber intake. Nile rats frequently nibble or crumble their food, and their cages are heavily littered with food waste every day, making it difficult to determine the amount of food consumed. The variations in daily fiber intake among different individual Nile rats can potentially affect the outcomes (e.g., diabetic risk). However, we expect that the overall trend should be robust given the large sample size in this study. 
In conclusion, we showed evidence that by improving early-life environment, such as using a maternal high-fiber diet, protective factors are transmitted to the offspring, leading to a remarkable reduction in the risk of susceptibility to type 2 diabetes.

Supplementary Materials: The following are available online at https:/ /www.mdpi.com/2072-664 3/13/1/94/s1, Figure S1: Graphic illustration of the number of Nile rats for each category, Figure S2: A simple Bayesian network for modeling relative diabetic risk, Figure S3: A high fiber offspring's diet can prevent to develop hyperglycemia regardless of maternal diet, Figure S4: The weight is heavier in offspring with maternal diabetogenic diet than with maternal high fiber diet at the time of weaning, Table S1: Detailed information of Nile rats used in this study.

Author Contributions: J.A.T. and H.T. designed the study; H.T. acquired the data; P.J. analyzed and interpreted data; H.T. and P.J. wrote the manuscript. All authors revised the manuscript and approved its final version. P.J. is the guarantor of this work. All authors have read and agreed to the published version of the manuscript.

Funding: This study was supported by the Garland Initiative for Vision funded by the William K. Bowes Jr. Foundation.

Institutional Review Board Statement: All animal experiments were approved by the University of California Santa Barbara, Institutional Animal Care and Use Committee, and conducted in accord with the NIH Guide for the Care and Use of Laboratory Animals.

Informed Consent Statement: Not applicable.

Data Availability Statement: The data that support the findings of this study are openly available in Supplementary Data.

Acknowledgments: The authors thank the K.C. Hayes (Brandeis University) for valuable advice on Nile rat husbandry and nutrition, and A. Freitag (Morgridge Institute for Research) for editorial assistance.

Conflicts of Interest: The authors declare no conflict of interest. The funders had no role in the design of the study; in the collection, analyses, or interpretation of data; or in the writing of the manuscript.

\section{References}

1. IDF Diabetes Atlas. Available online: https://www.diabetesatlas.org (accessed on 30 December 2020).

2. Taylor, K.S.; Heneghan, C.J.; Farmer, A.J.; Fuller, A.M.; Adler, A.I.; Aronson, J.K.; Stevens, R.J. All-Cause and Cardiovascular Mortality in Middle-Aged People with Type 2 Diabetes Compared with People without Diabetes in a Large UK Primary Care Database. Diabetes Care 2013, 36, 2366-2371. [CrossRef] [PubMed]

3. Meigs, J.B.; Cupples, L.A.; Wilson, P.W. Parental transmission of type 2 diabetes: The Framingham Offspring Study. Diabetes 2000, 49, 2201-2207. [CrossRef] [PubMed]

4. Fuchsberger, C.; Flannick, J.; Teslovich, T.M.; Mahajan, A.; Agarwala, V.; Gaulton, K.J.; Ma, C.; Fontanillas, P.; Moutsianas, L.; McCarthy, D.J.; et al. The genetic architecture of type 2 diabetes. Nature 2016, 536, 41-47. [CrossRef] [PubMed]

5. Prasad, R.B.; Groop, L. Genetics of Type 2 Diabetes-Pitfalls and Possibilities. Genes 2015, 6, 87-123. [CrossRef] [PubMed]

6. McNaughton, S.A.; Mishra, G.D.; Brunner, E.J. Dietary patterns, insulin resistance, and incidence of type 2 diabetes in the Whitehall II Study. Diabetes Care 2008, 31, 1343-1348. [CrossRef]

7. Odegaard, A.O.; Koh, W.P.; Butler, L.M.; Duval, S.; Gross, M.D.; Yu, M.C.; Yuan, J.M.; Pereira, M.A. Dietary patterns and incident type 2 diabetes in Chinese men and women: The Singapore Chinese health study. Diabetes Care 2011, 34, 880-885. [CrossRef]

8. Gluckman, P.D.; Cutfield, W.; Hofman, P.; Hanson, M.A. The fetal, neonatal, and infant environments-the long-term consequences for disease risk. Early Hum. Dev. 2005, 81, 51-59. [CrossRef]

9. Tay, J.; Thompson, C.H.; Luscombe-Marsh, N.D.; Wycherley, T.P.; Noakes, M.; Buckley, J.D.; Wittert, G.A.; Yancy, W.S., Jr.; Brinkworth, G.D. Effects of an energy-restricted low-carbohydrate, high unsaturated fat/low saturated fat diet versus a highcarbohydrate, low-fat diet in type 2 diabetes: A 2-year randomized clinical trial. Diabetes Obes. Metab. $2018,20,858-871$. [CrossRef]

10. van Zuuren, E.J.; Fedorowicz, Z.; Kuijpers, T.; Pijl, H. Effects of low-carbohydrate- compared with low-fat-diet interventions on metabolic control in people with type 2 diabetes: A systematic review including GRADE assessments. Am. J. Clin. Nutr. 2018, 108, 300-331. [CrossRef]

11. Weickert, M.O.; Pfeiffer, A.F.H. Impact of Dietary Fiber Consumption on Insulin Resistance and the Prevention of Type 2 Diabetes. J. Nutr. 2018, 148, 7-12. [CrossRef]

12. Hales, C.N.; Barker, D.J.P.; Clark, P.M.S.; Cox, L.J.; Fall, C.; Osmond, C.; Winter, P.D. Fetal and Infant Growth and Impaired Glucose-Tolerance at Age 64. BMJ-Br. Med. J. 1991, 303, 1019-1022. [CrossRef] [PubMed] 
13. Hales, C.N.; Barker, D.J. Type 2 (non-insulin-dependent) diabetes mellitus: The thrifty phenotype hypothesis. Diabetologia 1992, 35, 595-601. [CrossRef] [PubMed]

14. Schulz, L.C. The Dutch Hunger Winter and the developmental origins of health and disease. Proc. Natl. Acad. Sci. USA 2010, 107, 16757-16758. [CrossRef] [PubMed]

15. Bateson, P.; Gluckman, P.; Hanson, M. The biology of developmental plasticity and the Predictive Adaptive Response hypothesis J. Physiol. 2014, 592, 2357-2368. [CrossRef] [PubMed]

16. Petry, C.J.; Dorling, M.W.; Pawlak, D.B.; Ozanne, S.E.; Hales, C.N. Diabetes in old male offspring of rat dams fed a reduced protein diet. Int. J. Exp. Diabetes Res. 2001, 2, 139-143. [CrossRef] [PubMed]

17. Swanson, A.M.; David, A.L. Animal models of fetal growth restriction: Considerations for translational medicine. Placenta 2015, 36, 623-630. [CrossRef]

18. Zambrano, E.; Bautista, C.J.; Deas, M.; Martinez-Samayoa, P.M.; Gonzalez-Zamorano, M.; Ledesma, H.; Morales, J.; Larrea, F.; Nathanielsz, P.W. A low maternal protein diet during pregnancy and lactation has sex- and window of exposure-specific effects on offspring growth and food intake, glucose metabolism and serum leptin in the rat. J. Physiol.-Lond. 2006, 571, 221-230. [CrossRef]

19. Kereliuk, S.M.; Brawerman, G.M.; Dolinsky, V.W. Maternal Macronutrient Consumption and the Developmental Origins of Metabolic Disease in the Offspring. Int. J. Mol. Sci. 2017, 18, 1451. [CrossRef]

20. Yang, K.; Gotzmann, J.; Kuny, S.; Huang, H.; Sauve, Y.; Chan, C.B. Five stages of progressive beta-cell dysfunction in the laboratory Nile rat model of type 2 diabetes. J. Endocrinol. 2016, 229, 343-356. [CrossRef]

21. Subramaniam, A.; Landstrom, M.; Luu, A.; Hayes, K.C. The Nile Rat (Arvicanthis niloticus) as a Superior Carbohydrate-Sensitive Model for Type 2 Diabetes Mellitus (T2DM). Nutrients 2018, 10, 235. [CrossRef]

22. Chaabo, F.; Pronczuk, A.; Maslova, E.; Hayes, K. Nutritional correlates and dynamics of diabetes in the Nile rat (Arvicanthis niloticus): A novel model for diet-induced type 2 diabetes and the metabolic syndrome. Nutr. Metab. (Lond.) 2010, 7, 29. [CrossRef] [PubMed]

23. Subramaniam, A.; Landstrom, M.; Hayes, K.C. Genetic Permissiveness and Dietary Glycemic Load Interact to Predict Type-II Diabetes in the Nile rat (Arvicanthis niloticus). Nutrients 2019, 11, 1538. [CrossRef] [PubMed]

24. Bolsinger, J.; Landstrom, M.; Pronczuk, A.; Auerbach, A.; Hayes, K.C. Low glycemic load diets protect against metabolic syndrome and Type 2 diabetes mellitus in the male Nile rat. J. Nutr. Biochem. 2017, 42, 134-148. [CrossRef]

25. Ferland-McCollough, D.; Fernandez-Twinn, D.S.; Cannell, I.G.; David, H.; Warner, M.; Vaag, A.A.; Bork-Jensen, J.; Brons, C.; Gant, T.W.; Willis, A.E.; et al. Programming of adipose tissue miR-483-3p and GDF-3 expression by maternal diet in type 2 diabetes. Cell Death Differ. 2012, 19, 1003-1012. [CrossRef] [PubMed]

26. Bertram, C.; Trowern, A.R.; Copin, N.; Jackson, A.A.; Whorwood, C.B. The maternal diet during pregnancy programs altered expression of the glucocorticoid receptor and type 2 11beta-hydroxysteroid dehydrogenase: Potential molecular mechanisms underlying the programming of hypertension in utero. Endocrinology 2001, 142, 2841-2853. [CrossRef] [PubMed]

27. Geer, E.B.; Shen, W. Gender differences in insulin resistance, body composition, and energy balance. Gend. Med. 2009, 6 (Suppl. 1), 60-75. [CrossRef]

28. Logue, J.; Walker, J.J.; Colhoun, H.M.; Leese, G.P.; Lindsay, R.S.; McKnight, J.A.; Morris, A.D.; Pearson, D.W.; Petrie, J.R.; Philip, S.; et al. Do men develop type 2 diabetes at lower body mass indices than women? Diabetologia 2011, 54, 3003-3006. [CrossRef]

29. Needham, C.J.; Bradford, J.R.; Bulpitt, A.J.; Westhead, D.R. A primer on learning in Bayesian networks for computational biology. PLoS Comput. Biol. 2007, 3, e129. [CrossRef]

30. Scutari, M. Learning Bayesian Networks with the bnlearn R Package. J Stat. Softw. 2010, 35. [CrossRef]

31. Hagan, C. When are Mice Considered Old? Available online: https://www.jax.org/news-and-insights/jax-blog/2017 / november/when-are-mice-considered-old\# (accessed on 6 November 2017).

32. Wirstrom, T.; Hilding, A.; Gu, H.F.; Ostenson, C.G.; Bjorklund, A. Consumption of whole grain reduces risk of deteriorating glucose tolerance, including progression to prediabetes. Am. J. Clin. Nutr. 2013, 97, 179-187. [CrossRef]

33. Reynolds, A.; Mann, J.; Cummings, J.; Winter, N.; Mete, E.; Te Morenga, L. Carbohydrate quality and human health: A series of systematic reviews and meta-analyses. Lancet 2019, 393, 434-445. [CrossRef]

34. Zhao, L.; Zhang, F.; Ding, X.; Wu, G.; Lam, Y.Y.; Wang, X.; Fu, H.; Xue, X.; Lu, C.; Ma, J.; et al. Gut bacteria selectively promoted by dietary fibers alleviate type 2 diabetes. Science 2018, 359, 1151-1156. [CrossRef]

35. Vaiserman, A.M. Early-Life Nutritional Programming of Type 2 Diabetes: Experimental and Quasi-Experimental Evidence. Nutrients 2017, 9, 236. [CrossRef] [PubMed]

36. Hayward, A.D.; Lummaa, V. Testing the evolutionary basis of the predictive adaptive response hypothesis in a preindustrial human population. Evol. Med. Public Health 2013, 2013, 106-117. [CrossRef] [PubMed] 